\title{
The coming of age of EvoMPMI: evolutionary molecular plant-microbe interactions across multiple timescales
}

\author{
Jessica L Upson ${ }^{1 *}$, Erin K Zess ${ }^{1 *}$, Aleksandra Białas ${ }^{1 *}$, Chih-hang $\mathrm{Wu}^{1}$, Sophien Kamoun ${ }^{1}{ }^{\dagger}$ \\ ${ }^{1}$ The Sainsbury Laboratory, Norwich Research Park, Norwich, UK \\ * These authors contributed equally to this work \\ † Correspondence to: sophien.kamoun@tsl.ac.uk
}

\begin{abstract}
Plant-microbe interactions are great model systems to study co-evolutionary dynamics across multiple timescales, ranging from multimillion year macroevolution to extremely rapid evolutionary adaptations. However, mechanistic research on plant-microbe interactions has often been conducted with little consideration of the insights that can be gained from evolutionary concepts and methods. Conversely, evolutionary research has rarely integrated the diverse range of molecular mechanisms and models that continue to emerge from the molecular plant-microbe interactions field. These trends are changing. In recent years, the incipient field of evolutionary molecular plant-microbe interactions (EvoMPMI) has emerged to bridge the gap between mechanistic molecular research and evolutionary approaches. Here, we report on some of the recent advances in EvoMPMI. In particular, we highlight new systems to study microbe interactions with early diverging land plants, and new findings from studies of adaptive evolution in pathogens and plants. By linking mechanistic and evolutionary research, EvoMPMI promises to add a new dimension to our understanding of plant-microbe interactions.
\end{abstract}

\section{Introduction}

The field of plant-microbe interactions is remarkable for the recurrence of common processes across unrelated species and pathosystems. For example, distantly related plant pathogens exhibit extensive similarities in both their virulence and adaptation mechanisms, and conserved disease resistance toolkits and networks underlie immune responses across plant taxa. These features make plant-microbe interactions excellent model systems for developing and challenging evolutionary biology concepts.

Plant-microbe systems are also exceptional in that their evolutionary dynamics can be studied across multiple timescales. In macroevolutionary terms, microbes have shaped the evolution of plants since even before their arrival on land-and vice versa-resulting in fine-tuned interactions, specialised genome architectures, and increased robustness through redundancy and the emergence of networks. With shorter timescales in mind, the arms race that exists between plants and pathogens creates a tight interplay, with the plant activating defence responses that hinder the growth of the pathogen, whilst microbes deploy specialised molecular weapons that act within the plant to provide a foothold for infection. These dynamics create strong selective pressures, and have led to striking examples of rapid adaptive evolutionary change.

Nevertheless, mechanistic research on plant-microbe interactions has often been conducted without an appreciation of the insights that can be gained from evolutionary concepts and methods. Conversely, evolutionary research has rarely integrated the rich spectrum of molecular mechanisms and models that continue to emerge in the molecular plant-microbe interactions field. This is changing. In recent years, the nascent field of evolutionary molecular plant-microbe interactions (EvoMPMI) has emerged to link mechanistic molecular research to evolutionary approaches. We coined this acronym by analogy to EvoDevo to galvanize the community and highlight the emergence of this field of research. 
In this review, we summarise recent advances in EvoMPMI, with particular attention to plant-pathogen interactions. We broadly report on the trends and constraints that drive the coevolution of plants and microbes. In particular, we highlight new systems to study microbe interactions with early diverging land plants, and new findings from studies of adaptive evolution in pathogens and plants. By narrowing the gap between mechanistic and evolutionary research, EvoMPMI promises to add a new dimension to our understanding of plant-microbe interactions.

\section{The dawn of plant-microbe interactions}

Without microbes, plants, as we know them, would not exist. Terrestrial plants are thought to have evolved as the result of an ancient symbiosis between a semi-aquatic green alga and an aquatic fungus, borne onto land over 450 millennia ago [1]. In this model, the colonization of land by plants-and therefore their very evolution-was only possible through an intimate partnership with a filamentous microorganism [2-4].

Interestingly, this foundational fungal symbiosis may have come at a cost, since some microbes appear to have co-opted plant's symbiont accommodation to establish pathogenic interactions [4]. Indeed, some of the genes required for fungal symbiosis in plants are necessary for extensive colonization by biotrophic pathogens $[3,4]$. However, the topic appears to be somewhat controversial [5]. Nonetheless, analyses of the fossil record indicate that algae-pathogen and plant-pathogen interactions may date as far back as 400 million years ago (Mya); these consist of structures diagnostic of defence, such as enlarged cells and encasements around intracellular hyphae, respectively [6,7]. Moreover, a seed fern fossil that dates back to the Carboniferous period (300 Mya) displays a haustorium-like infection structure and is possibly the earliest evidence of parasitism by oomycetes [8].

Although there is evidence that plant-pathogen coevolution dates back to $\sim 400$ Mya, the cellular and molecular mechanisms underpinning these early interactions remain obscure. One approach that has gained popularity in recent years is to study the interactions of extant microbes with early diverging land plants, notably the bryophytes-hornworts, mosses, and liverworts-and determine how these interactions differ from those with angiosperms (Figure 1a) [9]. The rationale is that comparative studies with a range of land plants will shed light on ancestral features of plant-microbe interactions. It turns out that many microbial pathogens can infect bryophytes, causing disease symptoms such as necrosis and tissue maceration [2]. The oomycete Phytophthora palmivora was shown to colonize the photosynthetic layer of liverworts - the earliest diverging land plant-and to form intracellular hyphal structures similar to the haustoria produced on angiosperms [3] (Figure 1b). Liverwort cellular trafficking proteins and membrane protein syntaxins accumulate at these $P$. palmivora infection structures, suggesting that similar pathways are hijacked in all land plants [3]. Other studies have examined the moss Physcomitrella patens response to pathogen molecular patterns and filamentous microorganisms to gain insight into the evolution of plant defences $[2,10,11]$. $P$. patens perceives the presence of pathogens and activates defences that include the accumulation of reactive oxygen species, localized cell death, and induction of defence gene expression [10].

These experimental systems provide a range of exciting new platforms to study the evolutionary dynamics of plant susceptibility and immunity to microbial pathogens, and complement similar systems developed for symbiotic interactions [12]. We can now ask pertinent questions about the macroevolutionary dynamics of plant symbiosis, pathogenesis and immunity. How did microbes evolve to infect land plants? What are the commonalities and differences in microbial accommodation by plants across symbiotic and pathogenic interactions? When and how did plant immune receptors evolve? How did immune signalling pathways develop over time? This comparative approach-combined with detailed mechanistic understanding of microbial interactions with angiosperms-will shed light on the factors that have shaped these complex interactions and thus driven evolution. 


\section{Pathogen adaptations}

Plant hosts are continuously shaping pathogen evolution, driving recurrent and sustained changes within microbial genomes [13-15]. The push and pull of the natural selection forces imposed by host plants drive rapid and dynamic changes in the pathogen, ultimately shaping their genome evolution over both short and long timescales [15]. Successful infection requires the pathogen to modulate plant processes, for example by suppressing plant immunity. This is achieved via the actions of pathogen-secreted virulence factors known as effectors that act directly on host molecules [16-18]. Thus, pathogen adaptation to their host environment is intimately linked to the evolution of their effector repertoires. Remarkably, despite the huge diversity of plant pathogen taxa and the host species that they infect, the evolution of pathogen effectors shares many common features across diverse pathosystems.

What drives pathogen effector evolution? Two broad pressures imposed by the plant host have been defined: effectors evolve to adapt to host targets, or to evade detection by plant immune receptors (Figure 2). The interplay between these dynamic selection pressures creates an inherently unsettled biotic environment for effectors, accelerating the tempo of effector evolution.

Avirulence (AVR) effectors that are detected by plant immune receptors represent remarkable examples of rapid evolutionary adaptations. Notably, stealthy effector variants that evade detection by the plant whilst retaining virulence function (Figure $\mathbf{2 b}$ ) can carry extreme signatures of adaptive evolution with an excess of nonsynonymous polymorphisms (amino acid replacements) [19]. Such is the case for the effector AVR-Pik of the rice blast fungus Magnaporthe oryzae, in which allelic variants only carry non-synonymous polymorphisms that map to the binding interface of the rice immune receptor Pik-1 [20,21]. Each of the four amino acid polymorphisms of AVR-Pik appear to be adaptive [20,21], and the lack of synonymous changes is a hallmark of extremely rapid evolution, most likely driven by an accelerated arms race with the Pik-1 receptor [19]. In addition, effectors also exhibit marked patterns of selection following host jumps, where there is extreme pressure to adapt to new host targets (Figure 2a). For example, orthologous protease inhibitor effectors from the Solanaceae-infecting Phytophthora infestans, and its sister species, Phytophthora mirabilis, which infects Mirabilis jalapa, have adapted and specialized to protease targets unique to their respective host plants [22] (Fig. 3a).

Effectors can evolve through a birth and death process, resulting in significant levels of presence/absence polymorphisms within pathogen populations (Figure $\mathbf{2 b}$ )[23]. The maintenance of complex and possibly redundant effector repertoires could prove beneficial for a pathogen population, given that AVR effectors constrain the pathogen host range and their loss could enable the pathogen to jump to new hosts. A recent study on the emergence of wheat blast in Brazil in the 1980s vividly illustrates this model, and highlights the importance of studying effector evolution [24]. Losses of the effectors PWT3 and PWT4 in M. oryzae strains that infect the grass species Lolium were sufficient to enable a jump to wheat, which carries the matching resistance genes Rwt3 and Rwt4 [24]. The effector losses likely occurred in a stepwise manner, as PWT4 is absent or non-functional in most of the wheat-infecting isolates, whereas only a subset of these isolates also lack PWT3. The authors proposed that the widespread cultivation of wheat cultivars that lack Rwt3 in Brazil prior to the emergence of wheat blast served as a stepping stone for the pathogen to become fully virulent on the majority of wheat cultivars through stepwise mutations in the effectors (Fig. 3b). Following the host jump, wheat blast emerged as a destructive and pandemic disease of wheat, having spread from South America to South Asia where it's viewed as a serious threat to food security [25].

In addition to changes in individual effector genes, global patterns of pathogen genome evolution have been noted and may be linked to macroevolutionary trends, such as a high potential for host jumps $[13,15]$. Independent lineages of biotrophic plant pathogenic fungi and oomycetes can have highly expanded, repeat- 
bloated genomes $[13,26,27]$. Unrelated pathogens, such as the oomycete $P$. infestans and the fungus Leptosphaeria maculans, have converged towards an unusual genome architecture, in which rapidly evolving genes, particularly effectors, are localized to repeat-rich, gene-sparse compartments that contrast with the repeat-poor, gene-dense parts of the genome that carry housekeeping and more conserved genes $[15,26,28,29]$. This "two-speed genome" architecture was proposed to accelerate adaptive effector evolution through multiple mechanisms $[13,15,28]$. For example, proximity of effector genes to transposable elements enhances the rate of evolution of these genes, and may even increase the propensity of these genes to get silenced-an alternative mechanism to evade host immunity (Figure 2b) [30]. Also, the bipartite genome architecture may facilitate coordinated upregulation of effector genes during infection, possibly through epigenetic modifications $[13,28]$. Indeed, an important research goal is to determine the mechanisms that underpin epigenetic variation in rapidly evolving "two-speed" plant pathogen genomes. Recently, Chen et al. showed that $6 \mathrm{~mA}$ methylation in Phytophthora genomes is more prevalent in gene-sparse regions of the genomes, implicating this epigenetic mark in adaptive evolution [31]. However, future work is needed to determine the degree to which DNA methylation and other epigenetic modifications contribute to global patterns of pathogen genome evolution.

Although biotrophic filamentous plant pathogens tend to display an uneven genome architecture with effector genes populating repeat-rich and gene-poor regions, there are many variations to this theme, including effector gene clustering and occurrence in telomeric regions and accessory chromosomes [13]. Recently, the genome of the barley powdery mildew fungus, Blumeria graminis, was reported to be an exception to the "two-speed genome" model. B. graminis has a large repeat-expanded genome with structural and genetic variation dispersed throughout the genome [32]. Remarkably, repetitive DNA expansions affect $B$. graminis core ortholog genes in addition to effector genes [32]. That such an extreme case of genome expansion is not deleterious to the fungus may reflect the extreme evolutionary trajectories that the host-pathogen arms race imposes on the pathogen genome evolution.

The patterns of genome and effector evolution discussed above may not just apply to pathogenic microbes, but could also be relevant to plant parasitic animals such as insects. A recent analysis of the genome and transcriptome of the broad host range green peach aphid Myzus persicae revealed that clonal individuals can colonise distantly related hosts through differential regulation of sets of clustered genes [33]. The mechanisms underlying this rapid transcriptional plasticity remain unknown, but could involve some form of epigenetic modification [33]. It will be particularly interesting to compare insect strategies for infection to those of microbial plant pathogens. New insights into the evolution of the Mp10 effector of the green peach aphid points to marked differences with microbial pathogen effectors. Whereas the majority of plant pathogen effectors have rapidly diversified with little sequence and functional conservation [34], Mp10 is relatively conserved across hemipteran insects and belongs to the widespread chemosensory protein 4 clade (CSP4) [35]. Remarkably, the immunosuppression activity of Mp10 evolved via gain-of-function mutations over 250 million years ago, prior to the divergence of plant-sucking insect species [35]. As more effectors are discovered in aphids and other plant parasitic insects, it will be interesting to determine whether they would exhibit similar evolutionary trends.

\section{Host adaptations}

In the coevolutionary waltz between pathogens and plants, pathogens also shape the evolution of their hosts, leaving marked footprints on plant genomes (Box 1). The highly adaptable effector repertoires of plant pathogens ultimately shape the plant immune system, leading in most cases to an extremely diverse immune receptor repertoire that confers robust disease resistance against the majority of plant pathogens. This is particularly evident for the nucleotide binding leucine-rich repeat (NLR) class of proteins - the intracellular immune receptors that detect pathogens and trigger an effective immune response [36,37]. NLRs are present in all land plants, including early diverging species [38]. Hundreds of millions of years of plant-pathogen coevolution are apparent in NLR gene repertoires, which vary considerably in number and structure. With the 
availability of high-quality plant genomes and advances in functional characterization of plant immunity, studies on NLR evolution and adaptation have re-emerged as active research topics.

The role of effectors in shaping the evolution of immune receptors is best illustrated by the recent discovery of non-canonical domains in NLRs. Although the majority of NLRs have a conserved modular architecture, a subset of them carry these uncoventional integrated domains (IDs) $[39,40]$. Examples of NLR-ID fusions include the rice Pik-1 and RGA5 proteins, which carry integrated HMA (RATX1) domains [21,41], rice Pii-2 with a NOI/RIN4 domain [42], and Arabidopsis RRS1 which carries a WRKY domain[43]. IDs are thought to be derived from effector-associated host proteins, which then act as baits for effector recognition within NLRs [42,4446]. The number and diversity of IDs that have been reported in plant NLRs is astounding, and points to a highly successful evolutionary strategy of receptor diversification $[39,40]$. Nevertheless, the mechanisms underpinning the emergence of NLR-ID fusions are poorly understood. Bailey et al. recently proposed that ectopic recombination is a major driver of domain integration in Poaceae [47]. A "major integration clade", which includes RGA5, Pi-ta and Rpg5, underwent repeated independent integration events in the same region of the NLR [47]. Relatedly, Brabham et al have recently shown that a member of Mla locus RGH2 was subjected to fusions at its $3^{\prime}$ end [48]. Although the majority of $R G H 2$ homologues carry an integrated Exo70 domain, there is large variation in domain structure among $R G H 2$ homologues, including presence/absence of integration, which may be indicative of balancing selection acting on the gene. Overall, these studies indicate that some NLR loci may be more prone to recombination that favours integration, and thus serve as hotspots for the emergence of novel NLR-IDs. More work is needed to determine the frequency and mechanisms that drive the evolution of NLR fusions, as well as the degree to which novel fusions are maintained in plant populations.

NLR expansion and neofunctionalisation is another mechanism that enables plants to keep up with pathogens. NLR genes are often located in gene clusters that can act as a reservoir of genetic diversity [49-51]. Examples of NLR genes that have duplicated include members of the highly divergent Mla powdery mildew fungus resistance locus in barley [52]. NLR gene expansion is driven by ectopic recombination, unequal crossing over and transposition [50,53,54]. For instance, a recent comparative genomics analysis revealed that retrotransposition may have facilitated NLR expansion in Solanaceae [55]. Among the NLRs they examined, tomato $I 2$, potato $R 3 a$ and pepper $L$ genes appear to have originated from a single gene by retrotransposition followed by divergent evolution in each plant lineage to respond to different pathogens [55]. Interestingly, NLR genes are often in close proximity to various types of transposons, and the number of NLR gene clusters positively correlates with transposable element density [56], raising the possibility that transposon-driven duplications may be common in plants [52,57]. It is fascinating that NLR gene distribution and linkage to repetitive elements appears to mirror patterns of effector gene localization in plant pathogen genomes[58] (see above).

Another emerging paradigm is that NLRs can function cooperatively to form genetic networks of varying complexity. NLRs pairs are functionally specialized, with a sensor NLR that perceives a pathogen effector requiring a helper NLR to activate immune signalling [59]. Interestingly, each of the NLR-IDs that have been characterized to date are genetically and functionally linked to a helper NLR $[19,44]$. It is possible that paired NLRs have an increased tolerance to integration of non-canonical domains given that the ID is less likely to perturb the function of an NLR pair than of a singleton. NLR proteins can also form sophisticated signalling networks. A clear example is the NRC network, which has greatly expanded in Solanaceae and related species (Box 1), and exhibits an architecture where sensor NLRs rely on a few genetically downstream NRCs (NLR required for cell death) to establish disease resistance [60]. The NRCs and their sensor mates are phylogenetically related and have most likely evolved from an NLR pair that dramatically expanded in asterid plants about 100 Mya. Interestingly, the sensor NLRs underwent a much more significant expansion compared to the NRCs, which have diversified at a slower pace possibly due to constraints imposed by downstream signalling components. Convergence of immune signalling on a few NRCs may have facilitated the capacity of sensor NLRs to neofunctionalise and keep up with rapidly evolving pathogens. In this model, the classic bow- 
tie architecture of the NRC signalling network may enable higher evolutionary plasticity, and is reminiscent of the networks of plant cell-surface immune receptors and animal Toll-like receptors $[61,62]$.

Is NLR diversity only driven by antagonistic coevolution with pathogens? There is good evidence that NLR diversification may lead to autoimmunity and other deleterious effects on plant fitness, thus restraining their evolutionary dynamics[63]. NLR autoimmunity could be due to incompatible intramolecular interactions or interactions with other host proteins, including other NLRs. For example, Arabidopsis NLR proteins DM1 and DM2d trigger spontaneous cell death and hybrid necrosis when expressed in the same genetic background [64-66]. This indicates that the evolution of the plant immune system is not only shaped by pathogen selective pressures, but also by intrinsic constraints imposed by the genetic context. It is remarkable that NLR genetic incompatibility has been observed within populations of the same species, which highlights the extremely dynamic nature of NLR evolution. The high rate of pseudogenisation observed in NLR genes further points to potential fitness penalties associated with disease resistance $[56,67]$. In addition, overexpression of some NLRs can result in autoimmunity [68], and NLR gene expression is generally thought to be tightly regulated through chromatin modifications and transcription factors $[69,70]$. Furthermore, miRNAs may have also evolved to maintain homeostasis of expanded genes by targeting sequence-related NLR genes $[69,71]$. In summary, the evolutionary dynamics of plant NLRs are complex and are modulated by fitness trade-offs.

\section{Outlook: the coming of age of EvoMPMI}

We hope that the concepts and approaches discussed above will inspire further investigations that bridge the gap between evolutionary and mechanistic molecular research of plant-microbe interactions. Performing mechanistic research with an evolutionary perspective pushes the field of plant-microbe interactions beyond the molecular, laying the foundation to ask questions not only about how these systems function, but also about how they came to be that way. Many experimental systems would greatly benefit from comparative approaches performed within a phylogenetically and ecologically robust framework to test specific hypotheses about how evolution has tweaked mechanisms of pathogenicity, symbiosis, and immunity. This approach is especially robust whenever signals of adaptive evolution are detected. Such cases complement classical experimental approaches because they imply that natural selection has performed a genetic screen in the wild under relevant biological and ecological conditions. Thus, evolutionary approaches test mechanistic molecular models under real world scenarios bringing a certain degree of rigor to our understanding of plantmicrobe systems.

\section{Acknowledgements}

We are thankful to past and present members of the Kamoun Lab as well as to several colleagues for numerous discussions and ideas. We also thank lan Malcolm for his inspiration, and Russell Vance for a discussion that inspired Box 1. Apologies to colleagues whose work we didn't cite; unfortunately, we couldn't cover all relevant topics due to length restrictions and our focus on a few themes. AB receives funding from the Norwich Research Park, Doctoral Training Partnership (BBSRC, UK). JU receives funding from the Gatsby Charitable Foundation. Our laboratory is funded by the Gatsby Charitable Foundation, Biotechnology and Biological Sciences Research Council (BBSRC, UK), and European Research Council (ERC- NGRB and BLASTOFF). Follow research on evolutionary molecular plant-microbe interactions on Twitter using \#EvoMPMI.

\section{Figure Legends}

Figure 1. Specialized intracellular infection structures across land plants. (A) Evolution of microbial interactions in land plants. Simplified phylogeny of land plants with special attention to bryophytes (grey box) and estimated date of divergence (grey text). Evidence for specialized symbiotic or pathogenic intracellular infection structures in the various lineages is denoted by a ' + ,' whereas a '?' marks an interaction that has yet to be described $[73,74]$. The 
' $f$ ' denotes an interaction that has only been described in vitro [75]. (B) Intracellular pathogen infection structures across land plants. Intracellular infection structures of the Phytophthora species $P$. infestans and P. palmivora on evolutionarily distant hosts, the angiosperm Nicotiana benthamiana image reproduced from [72]) and the liverwort Marchantia polymorpha (image reproduced from [3]), respectively. Each Phytophthora species (red) forms a haustorium (marked by an arrow or star) - a digit-like protrusion that serves as the host interface-in its respective host cell, with cell boundaries indicated by cytoplasmic markers (top, turquoise) or plastid auto-fluorescence (bottom, turquoise).

Figure 2. Drivers of pathogen effector evolution. (A) Effectors adapt to new targets. Natural variation in an effector host target or changes in the pathogen biotic environment, for example following a host jump, drive effector adaptation. This results in effectors which can bind or act on a variant of the original target or on a totally new host target. (B) Effectors evade immune receptors. Effectors also evolve to evade recognition by host immune receptors. This can occur through adaptive mutations that result in stealthy effectors which avoid host recognition whilst retaining virulence activity. Alternatively, effector genes can also evade host immunity through pseudogenisation, deletion, or gene silencing.

Figure 3. Pathogen mutations associated with host jumps. (A) Adaptive mutations that accumulate after a host jump. Pathogens acquire mutations that fine-tune the pathogens interaction with its new host, such as in the case of the adaptive mutations in the Phytophthora mirabilis protease inhibitor effector PMEPIC, which arose to target a Mirabilis jalapa host protease [22]. Ultimately, such mutations lead to specialisation through inadvertent accumulation of mutations that are maladaptive in the ancestral host, in this case the Solanaceae. In this example, due to the relative distance of the evolutionary event, the causal mutations that enabled the host jump cannot be elucidated. (B) Mutations that enable a host jump. Serial loss of the Magnaporthe oryzae effectors PWT4 and PWT3 enabled the evolution of wheat-infecting lineages that are not constrained by the major determinants of wheat resistance, Rwt4 and Rwt3 [24]. Although these mutations are known to have occurred 35 years ago, the adaptive mutations that have subsequently accumulated in these lineages to fine-tune $M$. oryzae-wheat interaction are not yet known.

\section{Box Text}

\section{Box 1. Distinct evolutionary trends of NLR genes in mammals vs. plants.}

Although NLR genes are present both in mammalian and plant genomes, the evolutionary trajectories of their repertoires have been strikingly different. First, mammals have smaller NLR repertoires compared to plants. For instance, the human and mouse genomes carry 22 and 30 NLR genes, respectively, whereas in plants that number can range up to 402 in wheat $[36,76]$. Second, patterns of NLR evolution are different between mammals and plants across similar evolutionary timescales. For example, the divergence time for humans and mouse is estimated at around 80-100 Mya, similar to the divergence time between tomato and coffee. Nonetheless, despite this relatively long timescale, human (illustrated in yellow) and mouse (grey) NLRs are typically conserved with clear orthologous relationships $[77,78]$. In sharp contrast, there is usually little orthology among NLR genes across plant taxa such as tomato and coffee. For example, the NRC network of NLR genes has dramatically expanded in asterid plants from an NLR pair, with at least 47 members in tomato (red) and nearly 80 in coffee (green) scattered across the genomes of these species [60]. Accelerated evolution of the NRC network reflects massive functional diversification as the expanded genes confer resistance to pathogens and pests as divergent as viruses, bacteria, oomycetes, nematodes, aphids and whiteflies.

Why have mammalian and plant NLRs experienced strikingly different evolutionary trajectories across similar timescales? The answer may lie in the observation that NLRs from the plant and animal kingdoms tend to detect different types of pathogen molecules. Whereas mammalian NLRs typically detect fairly conserved pathogen molecular patterns (i.e. microbe-associated molecular patterns or MAMPs) [78], plant NLRs have evolved to detect pathogen and pest effectors that are delivered inside the host cell and are among the most rapidly evolving genes in microbial genomes. Thus, the evolutionary potential of the pathogens and their effectors drive the diversification of NLR genes, ultimately shaping the evolution of the host genome as noted with the dramatic expansions of NLR genes in plants [60]. 


\section{References}

1. Delaux P-M, Radhakrishnan GV, Jayaraman D, Cheema J, Malbreil M, Volkening JD, Sekimoto H, Nishiyama T, Melkonian M, Pokorny L, et al.: Algal ancestor of land plants was preadapted for symbiosis. Proc Natl Acad Sci 2015, 112:13390-13395.

2. Ponce de León I, Montesano M: Adaptation mechanisms in the evolution of moss defenses to microbes. Front Plant Sci 2017, 8:1-14.

3. Carella P, Gogleva A, Tomaselli M, Alfs C, Schornack S: Phytophthora palmivora establishes tissuespecific intracellular infection structures in the earliest divergent land plant lineage. bioRvix 2017.

4. Wang E, Schornack S, Marsh JF, Gobbato E, Schwessinger B, Eastmond P, Schultze M, Kamoun S, Oldroyd GED: A common signaling process that promotes mycorrhizal and oomycete colonization of plants. Curr Biol 2012, 22:2242-2246.

5. Huisman R, Bouwmeester K, Brattinga M, Govers F, Bisseling T, Limpens E: Haustorium formation in Medicago truncatula roots infected by Phytophthora palmivora does not involve the common endosymbiotic program shared by Arbuscular Mycorrhizal fungi and Rhizobia. Mol Plant Microbe Interact 2015, 28:1271-1280.

6. Taylor TN, Krings M: Fossil microorganisms and land plants: associations and interactions. Symbiosis 2005, 40:119-135.

7. Krings M, Taylor TN, Hass H, Kerp H, Dotzler N, Hermsen EJ: Fungal endophytes in a 400-million-yrold land plant: Infection pathways, spatial distribution, and host responses. New Phytol 2007, 174:648-657.

8. Strullu-Derrien C, Wawrzyniak Z, Goral T, Kenrick P: Fungal colonization of the rooting system of the early land plant Asteroxylon mackiei from the 407-Myr-old Rhynie Chert (Scotland, UK). Bot J Linn Soc 2015, 179:201-213.

9. Rensing SA, Lang D, Zimmer AD, Terry A, Salamov A, Shapiro H, Nishiyama T, Perroud P-F, Lindquist E a, Kamisugi $Y$, et al.: The Physcomitrella genome reveals evolutionary insights into the conquest of land by plants. Science 2008, 319:64-69.

10. Bressendorff S, Azevedo R, Kenchappa CS, Ponce de León I, Olsen J V., Rasmussen MW, Erbs G, Newman M-A, Petersen M, Mundy J: An innate immunity pathway in the moss Physcomitrella patens. Plant Cell 2016, 28:1328-1342.

11. Overdijk EJR, De Keijzer J, De Groot D, Schoina C, Bouwmeester K, Ketelaar T, Govers F: Interaction between the moss Physcomitrella patens and Phytophthora: a novel pathosystem for live-cell imaging of subcellular defence. J Microsc 2016, 263:171-180.

12. Ligrone R, Carafa A, Lumini E, Bianciotto V, Bonfante P, Duckett JG: Glomeromycotean associations in liverworts: A molecular, cellular, and taxonomic analysis. Am J Bot 2007, 94:1756-1777.

13. Raffaele $S$, Kamoun S: Genome evolution in filamentous plant pathogens: why bigger can be better. Nat Rev Microbiol 2012, 10:417-430.

14. Croll D, McDonald BA: The accessory genome as a cradle for adaptive evolution in pathogens. PLOS Pathog 2012, 8:8-10.

15. Dong S, Raffaele S, Kamoun S: The two-speed genomes of filamentous pathogens: Waltz with plants. Curr Opin Genet Dev 2015, 35:57-65.

16. Dodds PN, Rathjen JP: Plant immunity: towards an integrated view of plant-pathogen interactions. Nat Rev Genet 2010, 11:539-548.

17. Hogenhout SA, Van der Hoorn RAL, Terauchi R, Kamoun S: Emerging concepts in effector biology of plant-associated organisms. Mol Plant Microbe Interact 2009, 22:115-22.

18. Win J, Chaparro-Garcia , Belhaj K, Saunders DGO, Yoshida K, Dong S, Schornack S, Zipfel C, Robatzek $S$, Hogenhout $\mathrm{S}$ a, et al.: Effector biology of plant-associated organisms: concepts and perspectives. Cold Spring Harb Symp Quant Biol 2012, 77:235-47.

19. Białas A, Zess EK, De la Concepcion JC, Franceschetti M, Pennington HG, Yoshida K, Upson JL, Chanclud E, Wu C-H, Langner T, et al.: Lessons in effector and NLR biology of plant-microbe systems. Mol Plant-Microbe Interact 2017, 1:34-35. 
20. Yoshida K, Saitoh H, Fujisawa S, Kanzaki H, Matsumura H, Yoshida K, Tosa Y, Chuma I, Takano Y, Win J, et al.: Association genetics reveals three novel avirulence genes from the rice blast fungal pathogen Magnaporthe oryzae. Plant Cell 2009, 21:1573-91.

21. Maqbool A, Saitoh H, Franceschetti M, Stevenson C, Uemura a, Kanzaki H, Kamoun S, Terauchi R, Banfield M: Structural basis of pathogen recognition by an integrated HMA domain in a plant NLR immune receptor. Elife 2015, 4:1-24.

22. Dong S, Stam R, Cano LM, Song J, Sklenar J, Yoshida K, Bozkurt TO, Oliva R, Liu Z, Tian M, et al.: Effector Specialization in a Lineage of the Irish Potato Famine Pathogen. Science 2014, 343:552-555.

23. Fouché $S$, Plissonneau C, Croll D: The birth and death of effectors in rapidly evolving filamentous pathogen genomes. Curr Opin Microbiol 2018, 46:34-42.

24. Inoue Y, Vy TTP, Yoshida K, Asano H, Mitsuoka C, Asuke S, Anh VL, Cumagun CJR, Chuma I, Terauchi R, et al.: Evolution of the wheat blast fungus through functional losses in a host specificity determinant. Science 2017, 357:80-83.

25. Islam MT, Croll D, Gladieux P, Soanes DM, Persoons A, Bhattacharjee P, Hossain MS, Gupta DR, Rahman MM, Mahboob MG, et al.: Emergence of wheat blast in Bangladesh was caused by a South American lineage of Magnaporthe oryzae. BMC Biol 2016, 14:1-11.

26. Haas BJ, Kamoun S, Zody MC, Jiang RHY, Handsaker RE, Cano LM, Grabherr M, Kodira CD, Raffaele S, Torto-Alalibo T, et al.: Genome sequence and analysis of the Irish potato famine pathogen Phytophthora infestans. Nature 2009, 461:393-8.

27. Rouxel T, Grandaubert J, Hane JK, Hoede C, Van De Wouw AP, Couloux A, Dominguez V, Anthouard V, Bally $\mathrm{P}$, Bourras $\mathrm{S}$, et al.: Effector diversification within compartments of the Leptosphaeria maculans genome affected by repeat-induced point mutations. Nat Commun 2011, 2:202.

28. Soyer JL, El Ghalid M, Glaser N, Ollivier B, Linglin J, Grandaubert J, Balesdent MH, Connolly LR, Freitag $M$, Rouxel $T$, et al.: Epigenetic Ccontrol of effector gene expression in the plant pathogenic fungus Leptosphaeria maculans. PLoS Genet 2014, 10: e1004227.

29. Raffaele S, Farrer RA, Cano LM, Studholme DJ, MacLean D, Thines M, Jiang RHY, Zody MC, Kunjeti SG, Donofrio NM, et al.: Genome evolution following host jumps in the Irish Potato Famine pathogen lineage. Science 2010, 330:1540-1543.

30. Na R, Gijzen M: Escaping host immunity: new tricks for plant pathogens. PLoS Pathog 2016, 12:1-6.

31. Chen H, Shu H, Wang L, Zhang F, Li X, Ochola S, Mao F, Ma H, Ye W, Gu T, et al.: Phytophthora methylomes modulated by expanded $6 \mathrm{~mA}$ methyltransferases are associated with adaptive genome regions. bioRvix 2017.

32. Frantzeskakis L, Kracher B, Kusch S, Yoshikawa-maekawa M, Bauer S, Pedersen C, Spanu PD, Maekawa T, Schulze-lefert $P$, Panstruga R: Signatures of host specialization and a recent transposable element burst in the dynamic one-speed genome of the fungal barley powdery mildew pathogen. bioRxiv 2018.

33. Mathers TC, Chen Y, Kaithakottil G, Legeai F, Mugford ST, Baa-Puyoulet P, Bretaudeau A, Clavijo B, Colella S, Collin O, et al.: Rapid transcriptional plasticity of duplicated gene clusters enables a clonally reproducing aphid to colonise diverse plant species. Genome Biol 2017, 18:1-20.

34. Franceschetti M, Maqbool A, Jiménez-Dalmaroni MJ, Pennington HG, Kamoun S, Banfield MJ: Effectors of filamentous plant pathogens: commonalities amid diversity. Microbiol Mol Biol Rev 2017, 81:e00066-16.

35. Drurey C, Mathers TC, Prince DC, Wilson C, Caceres-Moreno C, Mugford ST, Hogenhout SA: Chemosensory proteins in the CSP4 clade evolved as plant immunity suppressors before two suborders of plant-feeding hemipteran insects diverged. bioRxiv 2017.

36. Jones JDG, Vance RE, Dangl JL: Intracellular innate immune surveillance devices in plants and animals. Science 2016, 354:aaf6395-aaf6395.

37. Kourelis J, van der Hoorn RAL: Defended to the Nines: 25 years of Resistance Gene Cloning Identifies Nine Mechanisms for R Protein Function. Plant Cell 2018.

38. Xue M, Yang J, Li Z, Hu S, Yao N, Dean RA, Zhao W, Shen M, Zhang H, Li C, et al.: Comparative analysis of the genomes of two field isolates of the rice blast fungus Magnaporthe oryzae. PLoS Genet 2012, 
8:e1002869.

39. Kroj T, Chanclud E, Michel-Romiti C, Grand X, Morel JB: Integration of decoy domains derived from protein targets of pathogen effectors into plant immune receptors is widespread. New Phytol 2016, 210:618-626.

40. Sarris PF, Cevik V, Dagdas G, Jones JDG, Krasileva K V: Comparative analysis of plant immune receptor architectures uncovers host proteins likely targeted by pathogens. BMC Biol 2016, 14:8.

41. Cesari S, Thilliez G, Ribot C, Chalvon V, Michel C, Jauneau A, Rivas S, Alaux L, Kanzaki H, Okuyama Y, et al.: The rice resistance protein pair RGA4/RGA5 recognizes the Magnaporthe oryzae effectors AVRPia and AVR1-CO39 by direct binding. Plant Cell 2013, 25:1463-1481.

42. Fujisaki K, Abe Y, Kanzaki E, Ito K, Utsushi H, Saitoh H, Białas A, Banfield MJ, Kamoun S, Terauchi R: An unconventional NOI/RIN4 domain of a rice NLR protein binds host EXO70 protein to confer fungal immunity. bioRxiv 2017.

43. Sarris PF, Duxbury Z, Huh SU, Ma Y, Segonzac C, Sklenar J, Derbyshire P, Cevik V, Rallapalli G, Saucet $\mathrm{SB}$, et al.: A plant immune receptor detects pathogen effectors that target WRKY transcription factors. Cell 2015, 161:1089-1100.

44. Cesari S, Bernoux M, Moncuquet P, Kroj T, Dodds PN: A novel conserved mechanism for plant NLR protein pairs: the "integrated decoy" hypothesis. Front Plant Sci 2014, 5:606.

45. Ellis JG: Integrated decoys and effector traps: how to catch a plant pathogen. BMC Biol 2016, 14:13.

46. Wu C-H, Krasileva K V., Banfield MJ, Terauchi R, Kamoun S: The "sensor domains" of plant NLR proteins: more than decoys? Front Plant Sci 2015, 6:5-7.

47. Bailey PC, Schudoma C, Jackson W, Baggs E, Dagdas G, Haerty W, Moscou M, Krasileva K V.: Dominant integration locus drives continuous diversification of plant immune receptors with exogenous domain fusions. bioRvix 2017.

48. Brabham HJ, Hernández-Pinzón I, Holden S, Lorang J, Moscou MJ: An ancient integration in a plant NLR is maintained as a trans-species polymorphism. bioRxiv 2017.

49. Jupe F, Pritchard L, Etherington GJ, MacKenzie K, Cock PJA, Wright F, Sharma SK, Bolser D, Bryan GJ, Jones JDG, et al.: Identification and localisation of the NB-LRR gene family within the potato genome. BMC Genomics 2012, 13:75.

50. Zhou T, Wang Y, Chen JQ, Araki H, Jing Z, Jiang K, Shen J, Tian D: Genome-wide identification of NBS genes in japonica rice reveals significant expansion of divergent non-TIR NBS-LRR genes. Mol Genet Genomics 2004, 271:402-415.

51. Meyers BC, Kaushik S, Nandety RS: Evolving disease resistance genes. Curr Opin Plant Biol 2005, 8:129-134.

52. Wei F, Wing R a, Wise RP: Genome dynamics and evolution of the Mla (powdery mildew) resistance locus in barley. Plant Cell 2002, 14:1903-1917.

53. Innes RW, Ameline-Torregrosa C, Ashfield T, Cannon E, Cannon SB, Chacko B, Chen NWG, Couloux A, Dalwani A, Denny R, et al.: Differential accumulation of retroelements and diversification of NB-LRR disease resistance genes in duplicated regions following polyploidy in the ancestor of soybean. Plant Physiol 2008, 148:1740-1759.

54. Kuang H: Multiple Genetic Processes Result in Heterogeneous Rates of Evolution within the Major Cluster Disease Resistance Genes in Lettuce. Plant Cell Online 2004, 16:2870-2894.

55. Kim S, Park J, Yeom S-I, Kim Y-M, Seo E, Kim K-T, Kim M-S, Lee JM, Cheong K, Shin H-S, et al.: Multiple reference genome sequences of hot pepper reveal the massive evolution of plant disease resistance genes by retroduplication. bioRxiv 2017.

56. Li J, Ding J, Zhang W, Zhang $Y$, Tang $P$, Chen JQ, Tian D, Yang S: Unique evolutionary pattern of numbers of gramineous NBS-LRR genes. Mol Genet Genomics 2010, 283:427-438.

57. Wicker T, Buchmann JP, Keller B: Patching gaps in plant genomes results in gene movement and erosion of colinearity. Genome Res 2010, 20:1229-1237.

58. Seidl MF, Thomma BPHJ: Transposable elements direct the coevolution between plants and microbes. Trends Genet 2017, 33:842-851.

59. Bonardi V, Tang SJ, Stallmann A, Roberts M, Cherkis K, Dangl JL: Expanded functions for a family of 
plant intracellular immune receptors beyond specific recognition of pathogen effectors. Proc NatI Acad Sci U S A 2011, 108:16463-16468.

60. Wu C-H, Abd-El-Haliem A, Bozkurt TO, Belhaj K, Terauchi R, Vossen JH, Kamoun S: NLR network mediates immunity to diverse plant pathogens. Proc Natl Acad Sci 2017, 114:201702041.

61. Oda K, Kitano H: A comprehensive map of the toll-like receptor signaling network. Mol Syst Biol 2006, 2.

62. Smakowska-Luzan E, Mott GA, Parys K, Stegmann M, Howton TC, Layeghifard M, Neuhold J, Lehner A, Kong J, Grünwald K, et al.: An extracellular network of Arabidopsis leucine-rich repeat receptor kinases. Nature 2018, 533:342-346.

63. Macqueen A, Sun X, Bergelson J: Genetic architecture and pleiotropy shape costs of Rps2-mediated resistance in Arabidopsis thaliana. Nat Plants 2016, 2: 16110.

64. Bomblies K, Lempe J, Epple P, Warthmann N, Lanz C, Dangl JL, Weigel D: Autoimmune response as a mechanism for a Dobzhansky-Muller-type incompatibility syndrome in plants. PLOS Biol 2007, 5:1962-1972.

65. Tran DTN, Chung E-H, Habring-Müller A, Demar M, Schwab R, Dangl JL, Weigel D, Chae E: Activation of a plant NLR complex through heteromeric association with an autoimmune risk variant of another NLR. Curr Biol 2017.

66. Chae E, Bomblies K, Kim S-T, Karelina D, Zaidem M, Ossowski S, Martín-Pizarro C, Laitinen RAE, Rowan $B A$, Tenenboim $\mathrm{H}$, et al.: Species-wide genetic incompatibility analysis identifies immune genes as hot spots of deleterious epistasis. Cell 2014, 159:1341-1351.

67. Marone D, Russo MA, Laidò G, De Leonardis AM, Mastrangelo AM: Plant nucleotide binding siteleucine-rich repeat (NBS-LRR) genes: Active guardians in host defense responses. Int J Mol Sci 2013, 14:7302-7326.

68. Heidrich K, Tsuda K, Blanvillain-Baufumé S, Wirthmueller L, Bautor J, Parker JE: Arabidopsis TNLWRKY domain receptor RRS1 contributes to temperature-conditioned RPS4 auto-immunity. Front Plant Sci 2013, 4:403.

69. Zhang $\mathrm{Y}$, Xia R, Kuang $\mathrm{H}$, Meyers $\mathrm{BC}$ : The diversification of plant NBS-LRR defense genes directs the evolution of microRNAs that target them. Mol Biol Evol 2016, 33:2692-2705.

70. Lai Y, Eulgem T: Transcript-level expression control of plant NLR genes. Mol Plant Pathol 2017.

71. De Vries S, Kloesges T, Rose LE: Evolutionarily dynamic, but robust, targeting of resistance genes by the miR482/2118 gene family in the Solanaceae. Genome Biol Evol 2015, 7:3307-3321.

72. Chaparro-Garcia A, Wilkinson RC, Gimenez-Ibanez S, Findlay K, Coffey MD, Zipfel C, Rathjen JP, Kamoun S, Schornack S: The receptor-like kinase SERK3/BAK1 is required for basal resistance against the late blight pathogen Phytophthora infestans in Nicotiana benthamiana. PLoS One 2011, 6.

73. Bonfante $P$ and Genre A.: Plants and arbuscular mycorrhizal fungi: an evolutionary-developmental perspective. Trends in Plant Science 2008, 13:1360.

74. Carella P and Schornack S.: Manipulation of bryophyte hosts by pathogenic and symbiotic microbes. Plant Cell Physiol 2017.

75. Hanke ST and Rensing SA.: In vitro association of non-seed plant gametophytes with arbuscular mycorrhiza fungi. Journal of Endocytobiosis and Cell Research 2010, 20:95.

76. Baggs E, Dagdas G, Krasileva K V.: NLR diversity, helpers and integrated domains: making sense of the NLR IDentity. Curr Opin Plant Biol 2017, 38:59-67.

77. Hibino T, Loza-Coll M, Messier C, Majeske AJ, Cohen AH, Terwilliger DP, Buckley KM, Brockton V, Nair $S \mathrm{~V}$., Berney K, et al.: The immune gene repertoire encoded in the purple sea urchin genome. DeV Biol 2006, 300:349-365.

78. Stein C, Caccamo M, Laird G, Leptin M: Conservation and divergence of gene families encoding components of innate immune response systems in zebrafish. Genome Biol 2007, 8: R251. 